\title{
Infarto del miocardio por picadura de abejas
}

\author{
Myocardial infarction due to bee stings
}

\author{
Guillermo Herrera, Boris Vesga, Carlos Rueda \\ - Bucaramanga (Colombia)
}

\section{Resumen}

El contacto con el veneno de abejas puede causar lesión miocárdica e incluso un infarto del miocardio propiamente dicho. Cuando esto ocurre en el contexto de reacción alérgica o anafilactoide se denomina síndrome de Kounis, una entidad subestimada que representa dilemas terapéuticos para el personal médico. Se recomienda tener un alto índice de sospecha en los casos de picaduras de abejas, para detectar oportunamente los pacientes que se benefician de terapia antisquémica, la cual se debe individualizar para evitar agravar la reacción inmune concomitante.

Palabras clave: himenóptero, infarto del miocardio, veneno de abejas

\begin{abstract}
Contact with bee venom can cause myocardial injury and even myocardial infarction itself. When this occurs in the context of an allergic or anaphylactoid reaction, it is called Kounis Syndrome, an underestimated entity that represents therapeutic dilemmas for medical personnel. It is recommended to have a high index of suspicion in cases of bee stings, to detect opportunely the patients that benefit from anti ischemic therapy, which must be individualized to avoid aggravating the concomitant immune reaction.
\end{abstract}

Key words: hymenoptera, myocardial infarction, bee venom
Dr. Guillermo Herrera: Departamento de Medicina Interna, Universidad Industrial de Santander; Dr. Boris Vesga: Docente Universidad Industrial de Santander. Instituto del Corazón de Bucaramanga; Dr. Carlos Rueda: Instituto del Corazón de Bucaramanga. Bucaramanga, (Colombia).

Correspondencia: Dr. Guillermo Herrera. Bucaramanga, (Colombia).

E-mail: guillermohrueda@hotmail.com

\section{Introducción}

Los ataques por himenópteros (abejas, avispas y hormigas) son la principal causa de muerte por emponzoñamiento en los Estados Unidos de América. La introducción activa de estos insectos dentro de la cadena de producción agrícola ha extendido su hábitat y el riesgo de contacto laboral o accidental con el veneno. En esta oportunidad se reporta un caso de ataque masivo de abejas africanizadas como desencadenante de un infarto agudo del miocardio con elevación del segmento ST (IAMCEST) rigurosamente documentado. Se discuten los dilemas terapéuticos que introduce la coexistencia fisiopatológica de infarto, toxicidad directa y respuesta inmune del emponzoñamiento masivo.

\section{Reporte de caso}

Una mujer de 52 años, obesa, con antecedente de diabetes mellitus tipo 2 de reciente diagnóstico con tratamiento farmacológico oral, sin otros antecedentes de importancia, fue llevada al servicio de urgencias tras ser víctima de un ataque masivo por abejas, mientras se encontraba en el área rural. En el examen físico se encontró álgica, somnolienta, taquicárdica y taquipneica. La auscultación cardiopulmonar fue normal, y en la piel se realizó un recuento de al menos
200 estigmas de picadura en rostro, cuero cabelludo, tórax y extremidades. Las lesiones cutáneas se caracterizaban por habones con algunas abejas y aguijones adheridos a los mismos (Figura 1A).

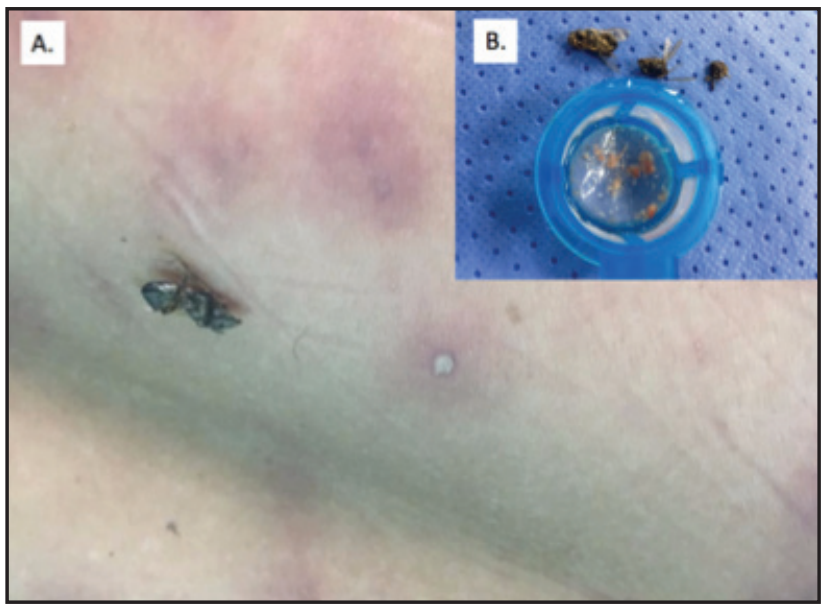

Figura 1.A. Fotografía del dorso de la paciente con lesiones y una abeja adherida a la piel. B. Fotografía de algunas de las abejas encontradas y elementos de la tromboaspiración. 
Después de ocho horas de observación en urgencias, la paciente presentó dolor epigástrico, disnea y diaforesis por lo cual fue remitida al cuarto nivel de complejidad. Se realizó electrocardiograma con hallazgos de elevación del segmento ST en las derivaciones precordiales V3 a V6. Considerándose un posible IAMCEST se indicó la realización inmediata de arteriografía que documentó la presencia de trombo que limitaba el flujo en el segmento proximal de la arteria descendente anterior y ocluía el segmento distal del mismo vaso. Se infundió heparina no fraccionada, inhibidor de glicoproteína IIb/IIIa (tirofibán) además de iniciar terapia antiplaquetaria dual. Pasadas 48 horas de evolución, se realizó angiografía de revisión, encontrando persistencia del trombo descrito, con mínima mejoría en la perfusión distal, por lo cual se optó por tromboaspiración mediante catéter (Figura 2). En la tomografía de coherencia óptica (OCT) no se evidenció la presencia de placa aterosclerótica complicada por ruptura o erosión, que predispusieran al evento trombótico.

La paciente evolucionó satisfactoriamente, el ecocardiograma de control mostró una función sistólica adecuada con fracción de eyección de 55\% y hubo mejoría en su clase funcional después del programa de rehabilitación cardiovascular.

\section{Discusión}

El veneno de abejas es una de las sustancias más sofisticadas de la naturaleza (1). Es una biotoxina con más de medio centenar de moléculas con capacidad inmunogénica y de lesión directa, entre las cuales se destaca la melitina $(60 \%)$ del contenido del veneno, un potente citotóxico vinculado en la injuria cardiaca reportada en varios casos de picaduras masivas de himenópteros (abejas, avispas y hor- migas) (2). No obstante, menos frecuentes son los reportes de verdaderos infartos agudos del miocardio con elevación del segmento ST como ocurre en este caso.

Se han descrito dos mecanismos fisiopatológicos para explicar el compromiso cardiaco después de un ataque por himenópteros (2). El primero implica haber tenido un contacto previo con los agentes inmunógenos contenidos en el veneno, que llevan al desarrollo de inmunidad celular y humoral que en un contacto posterior median la respuesta inmune que puede producir injuria miocárdica. Al respecto, en las últimas dos décadas se ha avanzado progresivamente en la comprensión de la antes llamada angina alérgica que hoy se denomina síndrome de Kounis (3) definido como la concurrencia de síndrome coronario con alergia, hipersensibilidad, anafilaxia o eventos anafilactoides. Los mecanismos específicos que inducen a injuria, isquemia o infarto en el contexto de una reacción inmune son variados pero resaltan la participación de mediadores celulares como la histamina y la triptasa, que pueden inducir vasoconstricción (vasoespasmo), procoagulabilidad y activación de la cascada inflamatoria en la placa ateromatosa e inestabilizarla. Por esta razón son susceptibles a sufrir de este síndrome, tanto pacientes con enfermedad coronaria previa, así como pacientes con coronarias anatómicamente sanas lo cual llevó a clasificar a esta entidad en tres categorías (4): síndrome de Kounis tipo I que incluye a los pacientes con anatomía coronaria normal sin factores predisponentes, en los cuales la exposición aguda a mediadores inflamatorios induce la aparición de espasmo. El tipo II describe individuos con lesiones ateromatosas preexistentes que se inestabilizan por la acción de mediadores inflamatorios produciendo erosión, ruptura y trombosis de la placa ateromatosa culpable y por

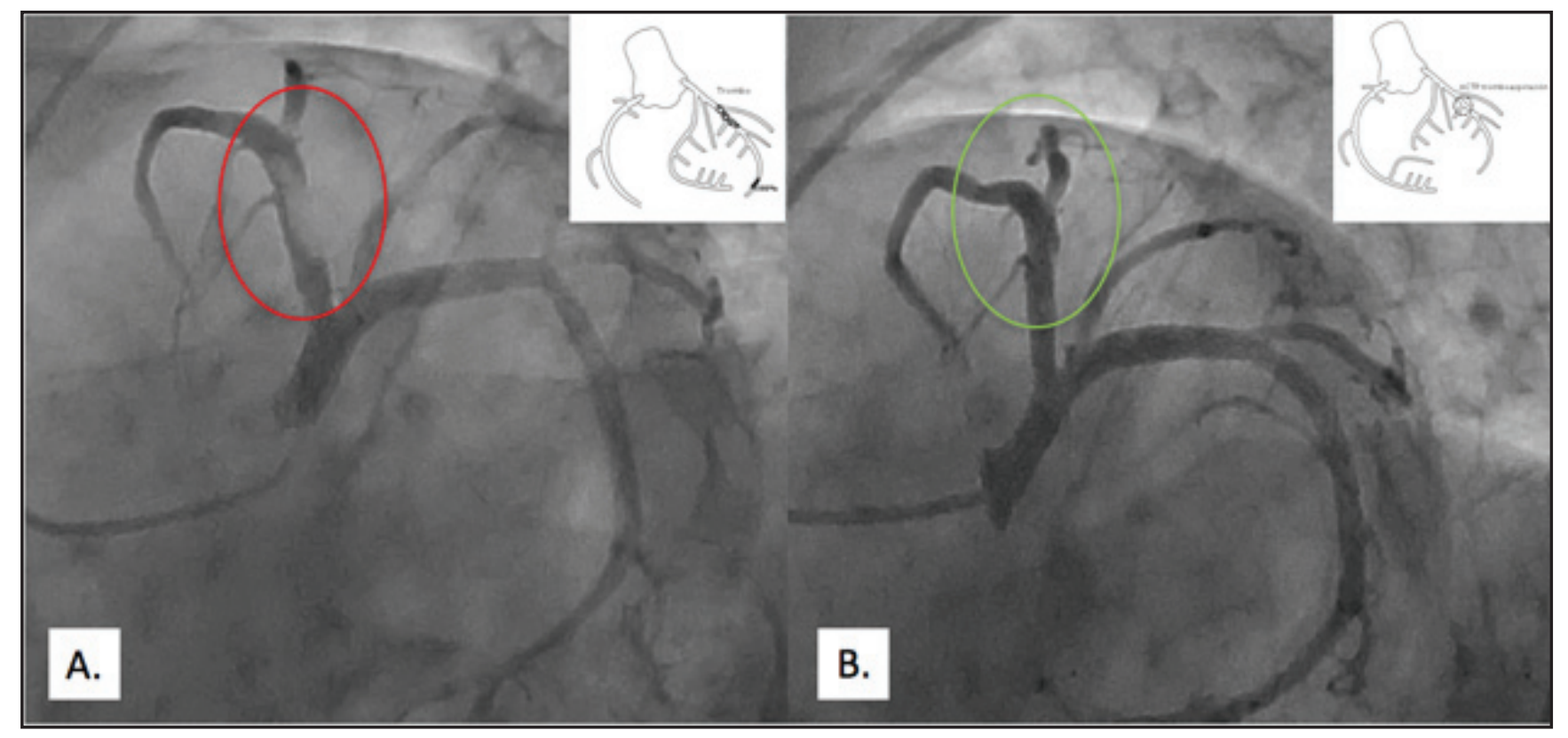

Figura 2.A. Arteriografía con evidencia de trombo en arteria descendente anterior. B. Imagen de la misma arteria después de trombo-aspiración evidenciándose la mejoría en la perfusión. 
último la variante tipo III que incluye casos de trombosis de stent coronario.

El segundo mecanismo fisiopatológico que explica el compromiso cardiaco por picadura de abejas es la lesión miocárdica directa por las toxinas del veneno, que a diferencia de las respuestas mediadas inmunológicamente tiene una relación dosis efecto; de hecho, ya se conoce la dosis letal 50 del veneno de abejas que corresponde a 1500 picaduras en un individuo de $75 \mathrm{~kg}$ o menos (5). Citotóxicos directos como la melitina producen lisis celular capaz de elevar los niveles sanguíneos de troponinas y así comprometer la función cardiaca como lo hacen otras sustancias orgánicas o inorgánicas descritas en la literatura, pero que por la rareza de los casos de ataques masivos no ha sido aclarado el efecto cardiaco de este tipo de veneno en humanos y la evidencia se limita a los datos que ofrecen modelos animales.

En este caso clínico, no es posible definir cuál es el impacto de cada uno de los dos mecanismos fisiopatológicos descritos, lo más probable es que ambos fenómenos sean concurrentes, porque a pesar de que la paciente no había tenido un contacto previo con picaduras de abejas que explicara una reacción inmunológica ante un segundo encuentro, el veneno de las abejas contiene histamina y factores estimuladores de la respuesta innata que podrían producir una respuesta anafilactoide desencadenando el infarto en un paciente susceptible.

Lamentablemente no existe un examen que permita hacer una confirmación específica para el diagnóstico del síndrome de Kounis. La medición de histamina es una herramienta propuesta desde el punto de vista teórico. Sin embargo, hay que tener en cuenta que tiene una vida media muy corta lo que limita su detección sérica (3). Al contrario, la triptasa es una biomolécula más estable, con más especificidad para la activación de mastocitos, siendo una herramienta útil para diferenciar eventos anafilácticos de los fenómenos anafilactoides $(4,6)$.

De esta manera, se insiste en que la ausencia de los marcadores descritos o carencia de historia de exposición evidente a un alérgeno no niegan la posibilidad de que el paciente presente un síndrome de Kounis (6) representing the American Academy of Allergy, Asthma \& Immunology (AAAAI).

La principal recomendación para el diagnóstico es tener un alto índice de sospecha y considerar la posibilidad de infarto del miocardio en los pacientes que han sufrido ataques por abejas. En este contexto deben incluirse el electrocardiograma, biomarcadores cardiacos y niveles de triptasa para documentar la coexistencia de reacción alérgica e infarto. Además, siempre que sea posible tomar muestra de los aspirados de trombos intrastent para buscar infiltración por eosinófilos (H\&E) y mastocitos (Giemsa), técnica que también se puede realizar en necropsias.

Las indicaciones para el tratamiento son controversiales porque se carece de evidencia para desarrollar guías (7). Entonces, se recurre a extrapolar las recomendaciones de expertos para cada una de las entidades por aparte: infarto y reacciones alérgicas. El resultado no se encuentra libre de dilemas, dado que se pueden encontrar francas contradicciones en el uso de medicamentos que podrían estar, al menos teóricamente, indicados y contraindicados en el paciente con infarto por picadura de abejas.

En general, se debe garantizar una adecuada oxigenación tisular a estos pacientes, en especial en casos de anafilaxia, incluso si es necesario instaurar una vía aérea estable con intubación orotraqueal.

Las reacciones alérgicas graves pueden provocar hipotensión arterial, condición que contraindica usar medicamentos vasodilatadores o antihipertensivos. Al respecto los nitratos, teóricamente pueden favorecer la liberación de histamina al igual que los opiáceos (3) y los inhibidores de la enzima convertidora de angiotensina (6) favorecen la vía de las bradicininas a favor de la respuesta alérgica, no obstante, estos efectos no suelen ser significativos y su uso está indicado siempre que la tensión arterial del paciente lo permita. Por su parte los antagonistas de los canales de calcio deben tenerse en cuenta como opción terapéutica porque tienen un papel importante en el tratamiento del infarto causado por vasoespasmo (4).

El ácido acetilsalicílico como antiagregante plaquetario debe usarse en todos los pacientes incluso ante la simple sospecha clínica de infarto, siempre que no haya contraindicación. Sin embargo, es uno de los medicamentos más asociados con hipersensibilidad, por lo cual siempre que se considere la posibilidad de que este medicamento pueda empeorar el proceso alérgico se recomienda desensibilizar o usar otras opciones antiplaquetarias como los antagonistas P2Y12 (por ejemplo: clopidogrel) (7).

La adrenalina es el tratamiento de elección para la anafilaxia (6), a pesar de que puede empeorar la isquemia por muchos mecanismos y debe usarse siempre que se requiera. Los betabloqueadores tienen varios efectos pleiotrópicos favorables para los pacientes que han sufrido infartos del miocardio, no obstante, en el contexto de alergia pueden oponerse al sistema simpático o a la adrenalina exógena y empeorar la reacción alérgica, broncoconstricción e hipotensión asociada.

Ante la hipotensión en el contexto de anafilaxia se debe hacer reposición de líquidos endovenosos, pero el riesgo de disfunción del ventrículo por el infarto se traduce en riesgo de edema pulmonar. Se sugiere ser cauteloso con la reposición de volumen manteniendo un monitoreo estricto del balance hídrico del paciente.

Los antihistamínicos parenterales son medicamentos útiles para el control de síntomas de las reacciones alérgicas, pero no son de primera línea en anafilaxia. En dicha circunstancia suelen usarse por vía parenteral con riesgo de efectos adversos cardiovasculares, como hipotensión. Así mismo los anti $\mathrm{H} 2$ se usan como protectores de mucosa gástrica que pueden tener función sinérgica en el control de los síntomas cutáneos de la alergia al usarse en conjunto de los anti H1, 
además en comparación de otras opciones como los inhibidores de la bomba de protones, no interfieren (teóricamente) con el metabolismo de fármacos de la terapia antiisquémica. Otro grupo de medicamentos de interés en la alergia son los estabilizadores de membrana de los mastocitos, que no han mostrado utilidad en el manejo de fenómenos alérgicos agudos. Por último los corticoesteroides pueden tener una participación más importante en los procesos alérgicos que los antihistamínicos, pero no son de primera línea en la anafilaxia, en especial porque su mecanismo de acción no es inmediato pero en últimas su uso sigue siendo recomendado por la mayoría de autores (8), sin olvidar que existe evidencia que sugiere que pueden inducir la remodelación de la pared miocárdica aumentando la susceptibilidad a complicaciones graves como aneurismas de la pared y su ruptura.

En conclusión, el efecto del veneno de abejas puede producir lesión miocárdica por medio de dos mecanismos: inmunológico y tóxico directo cuyo impacto no se puede determinar por separado. Así, por definición, un infarto del miocardio en el contexto de un proceso alérgico se configura como un síndrome de Kounis, el cual representa un reto terapéutico a la hora de conciliar la terapia antiisquémica y la antialérgica.

\section{Agradecimientos y declaraciones}

Los autores no tienen conflictos de interés, obtuvieron consentimiento informado de la paciente y agradecen al Departamento de Medicina Interna de la Universidad Industrial de Santander y al personal del servicio de hemodinamia del Instituto del Corazón de Bucaramanga por sus aportes en el análisis del caso.

\section{Referencias}

1. Gupta, Prabha Nini; Kumar, B Krishna; Velappan, Praveen; Sudheer MD Possible complication of bee stings and a review of the cardiac effects of bee stings. BMJ Case Rep. 2016; In press (May).

2. Almeida RAMDB, Olivo TET, Mendes RP, et al. Africanized honeybee stings: how to treat them. Rev Soc Bras Med Trop. 2011; 44 (6): 755-761. doi:10.1590/ S0037-86822011000600020.

3. Kounis NG. Kounis syndrome: An update on epidemiology, pathogenesis, diagnosis and therapeutic management. Clin Chem Lab Med. 2016; 54 (10): 1545-1559. doi:10.1515/cclm-2016-0010.

4. Rico Cepeda P, Palencia Herrejón E, Rodríguez Aguirregabiria MM Síndrome de Kounis. Med Intensiva. 2012; 36 (5): 358-364. doi:10.1016/j. medin.2011.10.008

5. Aminiahidashti H, Laali A, Samakoosh A, Gorji A. Myocardial infarction following a bee sting: A case report of Kounis syndrome. Ann Card Anaesth. 2016 19 (2): 375-378. doi:10.4103/0971-9784.179626.

6. Golden DBK, Demain J, Freeman T, et al. Stinging insect hypersensitivity: A practice parameter update 2016. Ann Allergy, Asthma Immunol. 2017; 118 (1): 28-54. doi:10.1016/j.anai.2016.10.031.

7. Villamil-Munévar PA, Sánchez-Solanilla LF. Síndrome de Kounis o angina alérgica. Rev Colomb Cardiol. 2017;. doi: 10.1016/j.rccar.2016.10.042.

8. Bonifazi F, Jutel M, Bilo BM, Birnbaum J, Muller U. Prevention and treatment of hymenoptera venom allergy: guidelines for clinical practice. Allergy. 2005; 60 (12): 1459-1470. doi:10.1111/j.1398-9995.2005.00960.x. 